\title{
Using Gene Expression Signatures to Dissect Insulin Resistance Subtypes
}

\author{
Brad Hayward, Nicky Konstantopoulos and Ken R. Walder \\ Metabolic Research Unit, School of Medicine, Deakin University \\ Australia
}

\section{Introduction}

It is now apparent that many diseases such as diabetes are more complex and heterogeneous than had been thought just a decade ago. Combinations of varying causative factors, as well as interactions between environmental and genetic factors all play a role in the onset of the disease. This complexity has hindered the development of new effective treatment options for patients, and makes understanding the onset of the disease difficult. This chapter will focus on a new technology to study diabetes using a novel unbiased approach, and to develop individualised therapeutics for patients with diabetes.

\subsection{Type 2 diabetes}

Diabetes mellitus is characterised by dysregulation of a number of metabolic processes as a result of abnormal insulin secretion and/or signalling (Saltiel and Pessin, 2002). Insulin, secreted by the pancreas, is a potent anabolic hormone involved in the regulation of glucose homeostasis as well as lipid and protein metabolism (Saltiel and Pessin, 2002). There are two main types of diabetes mellitus. Type 1 diabetes (T1D), is caused by a defect in insulin secretion by the pancreas, and can be treated by administration of exogenous insulin. T1D is often caused by an autoimmune disorder, where the insulin-secreting $\beta$-cells of the pancreas are destroyed, however, additional environmental causes such as viruses may also be involved (Tisch and McDevitt, 1996). In contrast, type 2 diabetes (T2D) is characterised by resistance to the action of insulin in key metabolic tissues such as skeletal muscle, liver and adipose tissue, coupled with reduced insulin secretion caused by impaired $\beta$-cell function in the pancreas (McKinlay and Marceau, 2000; NIDDK, 2009).

T2D accounts for over $90 \%$ of all reported cases of diabetes (Taylor, 1999). The disease is characterised by peripheral insulin resistance, hyperglycaemia and defective insulin secretion. Defective insulin signalling in peripheral tissues including muscle, adipose tissue and the liver, adversely affects whole body glucose homeostasis. Impaired insulin signalling, coupled with the eventual exhaustion of $\beta$-cell insulin production, leads to T2D (Fig. 1). Unlike type 1 diabetes, where insulin therapy can provide effective relief, T2D requires treatment of insulin resistance, in addition to insulin secretion defects. 


\section{Insulin Resistance}

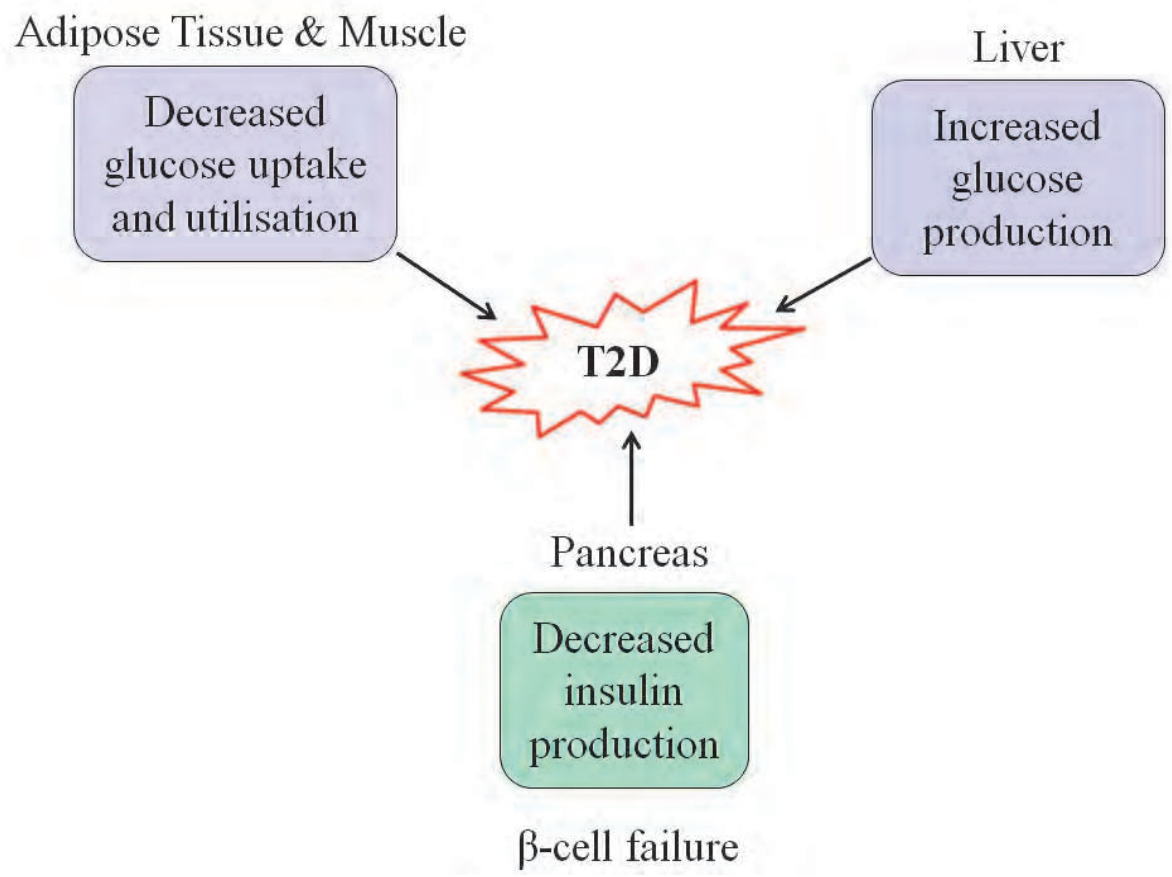

Fig. 1. A simplified overview of the pathogenesis of type 2 diabetes, encompassing insulin resistance in muscle, adipose tissue and liver, as well as impaired insulin secretion by the $\beta$ cells of the pancreas. Adapted from (Baudry et al., 2002).

\subsection{Prevalence and cost of diabetes}

There are currently 25.8 million people in the United States living with diabetes, and this accounts for $8.3 \%$ of the population (CDC, 2011). This alarming figure is growing rapidly, with 1.9 million people being newly diagnosed in 2010 alone (CDC, 2011). Diabetes represents a significant health burden to the US, both in terms of the number of patients currently living with diabetes, and the huge number of patients estimated to develop diabetes in the coming years. It has been estimated that there are currently 79 million adults in the US who are prediabetic (as determined by fasting blood glucose or HbA1c levels). The costs associated with managing the diabetes epidemic were recently estimated at $\$ 174$ billion annually, and this figure is set to increase in the coming years. The projected increase in the prevalence of diabetes, coupled with the already significant economic costs associated with the disease, make the development of alternative effective treatments an urgent priority.

\section{Diagnosis and treatment of type 2 diabetes}

\subsection{Diagnosis of type 2 diabetes}

Diagnosis of T2D, and its precursor insulin resistance, is made difficult by the lack of symptoms early in the development of the disease, and many cases go undiagnosed. The 
Australian Diabetes, Obesity and Lifestyle study found that half of all subjects studied who were suffering from T2D had not been previously diagnosed (Dunstan et al., 2002). Predictors of risk for the development of T2D and cardiovascular disease include body mass index (BMI), ethnic origin, blood pressure and cholesterol levels (Gavin et al., 2003). Current clinical guidelines for the diagnosis of diabetes however are based upon blood glucose measures. The World Health Organisation (WHO) standard criteria for diagnosis of T2D involve fasting plasma glucose (FPG) and the response to an oral glucose tolerance test (OGTT). FPG is a measure of plasma glucose after 8 hours of fasting, while the OGTT measures plasma glucose 2 hours following an intake of $75 \mathrm{~g}$ glucose. The current guidelines are outlined in Table 1.

\begin{tabular}{|lll|}
\hline & FPG & OGTT \\
\hline NGT & $<6.1 \mathrm{mmol} / \mathrm{L}$ & $<7.8 \mathrm{mmol} / \mathrm{L}$ \\
\hline IGT & $<7.0 \mathrm{mmol} / \mathrm{L}$ & $7.8-11.1 \mathrm{mmol} / \mathrm{L}$ \\
\hline IFG & $6.1-6.9 \mathrm{mmol} / \mathrm{L}$ & $<7.8 \mathrm{mmol} / \mathrm{L}$ \\
\hline Diabetes & $\geq 7.0 \mathrm{mmol} / \mathrm{L}$ & $\geq 11.1 \mathrm{mmol} / \mathrm{L}$ \\
\hline
\end{tabular}

Abbreviations used: NGT - normal glucose tolerance, IGT - impaired glucose tolerance, IFG - impaired fasting glucose (WHO/IDF, 2006).

Table 1. WHO guidelines for the diagnosis of diabetes and other hyperglycaemic states.

IGT and IFG are both strong indicators of risk for the development of T2D, with individuals suffering from both conditions placed at even higher risk (Gavin et al., 2003). IGT is characterised by peripheral insulin resistance, while defects in insulin secretion coupled with increased hepatic glucose output characterise IFG (Davies et al., 2000). While the associated health risks, prevalence and distribution vary for IGT and IFG (Unwin et al., 2002), the risk of developing T2D is similar for both, and increases further when both IGT and IFG are present (Gavin et al., 2003).

\subsection{Current anti-diabetic treatments}

The development of both insulin resistance and impaired glucose tolerance, conditions which precede the onset of T2D, are closely linked with obesity (Sharma, 2006). Excess visceral fat, and the hormones and inflammatory factors it releases, coupled with excess free fatty acid release have been implicated in the development of T2D (Mlinar et al., 2007). For obese patients exhibiting these symptoms, changes to healthier eating patterns and increases in exercise can result in improvements to glucose tolerance. However this approach often fails within the first year of treatment, and therefore the use of various medications is usually required (Nathan et al., 2006). Lifestyle changes immediately following the diagnosis of T2D can often be successful in the early treatment of the disease. Unfortunately, a lack of diagnosis, coupled with difficulties in maintaining lifestyle changes, means that this is not a treatment option which will be effective in the long term for all patients (Nathan et al., 2006).

Metformin is an oral antidiabetic agent, based upon the molecule biguanide. Its mechanism of action involves a reduction in hepatic gluconeogenesis, leading to a reduction in blood glucose levels (Knowler et al., 2002). This can also have the associated benefit of reducing blood insulin levels. Metformin has a number of side effects including gastrointestinal symptoms and has been linked with rare cases of lactic acidosis which can be fatal, although 
evidence for this has been contradicted in some studies (Salpeter et al., 2006). Metformin is one of only two oral anti-diabetic agents on the WHO list of essential medicines. The second oral anti-diabetic to be listed by WHO is the drug family known as the sulfonylureas, the most commonly used drug of which is glibenclamide. The sulfonylureas mechanism of action involves enhancing insulin secretion (Groop, 1992). For this reason, the sulfonylureas show their best efficacy in the early stages of the disease when $\beta$-cell function is still viable. Side effects associated with the sulfonylureas include hypoglycaemia due to their long half life in plasma, and weight gain.

The glinides are a family of drugs with a mechanism of action similar to the sulfonylureas, in that they bind to the same receptor - although at a different binding site - to induce insulin secretion from the $\beta$-cells of the pancreas. The glinides have an advantage over the sulfonylureas in that they have a shorter half life in blood plasma. As such, some glinides pose a lower risk of hypoglycaemia then some of the sulfonylureas (Kristensen et al., 2000). Thiazolidinediones (TZDs or glitazones) are an insulin sensitizing family of compounds. TZDs are ligands for the nuclear transcription factor peroxisome proliferator-activated receptor $Y$ (PPARY). It is through transcriptional regulation of PPARY that this family of compounds increase the sensitivity of muscle, liver and adipose tissue to the effects of insulin (Yki-Jarvinen, 2004). However, this family of drugs has been linked to some serious long term side effects. Troglitazone, first approved for use in T2D patients in 1997, was withdrawn from the market in 2000 after it was linked to a number of cases of liver dysfunction and failure (Watkins, 2005). The widely used alternative rosiglitazone has in recent years been linked to increased cardiovascular disease (Nissen and Wolski, 2010). The drug has been withdrawn from sale in the UK and New Zealand. While still available in the US, rosiglitazone is currently branded with additional safety warnings and restrictions on its use, and sales in recent years have fallen significantly (GlaxoSmithKline, 2010).

Exogenous insulin is a very important therapeutic agent for the treatment of diabetes, capable of increasing blood insulin levels when $\beta$-cell function has been impaired, and can be given in increasing amounts to overcome insulin resistance. However, insulin is also associated with increases in weight gain, as well as risk of hypoglycaemia if monitoring of blood glucose levels is not rigorously performed.

Glucagon-like peptide 1 agonists (GLP-1 agonists) are mimics of a protein secreted by the Lcells of the small intestine. They act on GLP-1 receptors in pancreatic $\beta$-cells, inducing insulin release. GLP-1 agonists have also been shown to stimulate $\beta$-cell proliferation (Drucker, 2003, 2005) and suppress glucagon release and gastric motility, while inducing weight loss. Side effects of GLP-1 agonists include a decrease in gastric motility, responsible for the nausea commonly experienced by patients (Kendall et al., 2005).

Amylin is a $\beta$-cell hormone co-secreted with insulin. Amylin lowers blood glucose levels by inhibiting glucagon secretion following a meal, and induces satiety by acting upon the area postrema (AP) neurons within the brain stem (Potes and Lutz, 2010). While amylin forms aggregates which make it unsuitable as a therapeutic agent, amylin agonists such as pramlintide can effectively simulate the effects of the physiological amylin. Like GLP-1 agonists, amylin agonists can also induce nausea in patients (Schmitz et al., 2004).

\subsection{Problems and adverse effects of current drug therapies}

As highlighted above, the currently used range of antidiabetic medicines have a number of adverse side effects, including hypoglycaemia, fluid retention and weight gain, and gastro- 
intestinal symptoms. As T2D generally progresses over time to a worsening in glycaemic control, the need to utilise multiple therapies together is unfortunately the reality for many patients with T2D (Nathan et al., 2006). Difficulties in managing T2D are exacerbated by the fact that the various drugs available have a wide range of effects in individual patients, in terms of the magnitude of both efficacy and side effects. In addition to these factors, many of the current drugs used to treat T2D lose their efficacy over time (Cohen and Horton, 2007). Therefore, the focus of new treatments has to be on how to personally tailor pharmacotherapy to suit each patient's characteristics.

We believe that the reason why current therapies are not effective in all patients is that they do not address the heterogeneous nature of T2D. A number of different subtypes of insulin resistance have been described, in a number of different tissues and due to varying insults. If effective treatments for T2D are to be developed, there is a need to gain a better understanding of the different subtypes of insulin resistance. Then, the development of new treatment regimes which specifically target the various subtypes of insulin resistance will be possible - enabling the development of a personalised medicine approach to T2D.

\section{Insulin resistance subtypes}

\subsection{Insulin resistance subtypes}

Insulin resistance is a major risk factor for the development of T2D (Lillioja et al., 1993). Combating insulin resistance is therefore a key to developing effective treatments for T2D. The etiology of T2D is multifactorial, with both genetic and environmental factors involved (Bergman and Ader, 2000). Likewise, the onset of insulin resistance is multifactorial and can occur in different tissues and arise from multiple causes as depicted in Fig. 2. There are numerous known insults to insulin signalling and action. Insults to insulin action can be both endogenous, such as inflammatory cytokines released in response to a fatty meal, and exogenous, such as the fatty acids themselves, which can lead to the development of insulin resistance. These subtypes can be mimicked in cell culture based models, as shown in Table 2 .

\begin{tabular}{ll}
\hline Subtype & Causative agents \\
\hline Inflammation & Cytokines: eg. Some interleukins, TNFa \\
\hline ER Stress & Tunicamycin, Thapsigargin \\
\hline Glucocorticoid & Dexamethasone \\
\hline Hyperinsulinemia & Chronic elevated insulin levels \\
\hline Oxidative stress & ROS: eg. Superoxide anions \\
\hline Hyperlipidemia & $\begin{array}{l}\text { Long chain, saturated FFAs: eg. Palmitate (16:0) (Chavez and } \\
\text { Summers, 2003) }\end{array}$ \\
\hline
\end{tabular}

Table 2. Proposed subtypes of insulin resistance and the insults which can lead to their genesis in cell models.

While there are a number of factors which may lead to the development of insulin resistance in various tissues, they do not necessarily develop in complete isolation, and signalling crosstalk between the various models mentioned above occurs. For example, hyperlipidemia induced insulin resistance has also been linked to increased generation of the inflammatory cytokine TNF- $\alpha$ through activation of proinflammatory transcription factor NF-k $\beta$ (Itani et al., 2002; Jove et al., 2006). 


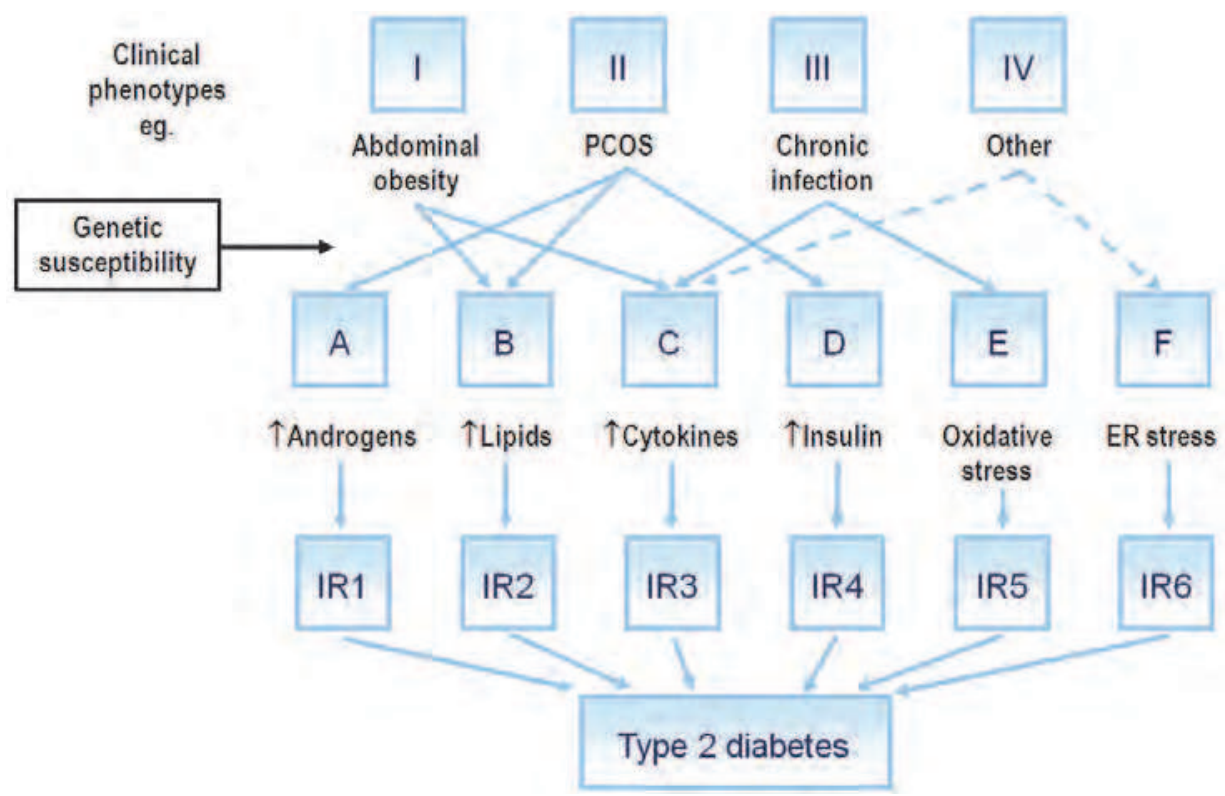

Fig. 2. The multifactorial nature of T2D. Multiple clinical phenotypes such as abdominal obesity, polycystic ovary (PCOS) and Cushing's syndromes, lipodystrophies, chronic levels of hyperinsulinemia, acromegaly (elevated growth hormone) and chronic infection are all associated with insulin resistance. The links between the causes of insulin resistance associated with these phenotypes is not obvious since multiple pathways have been implicated in the development of insulin resistance, such as hyperlipidemia, elevated levels of pro-inflammatory cytokines and/or induction of oxidative or endoplasmic reticulum (ER) stress pathways which may be activated individually or concurrently. Superimposed on this network of interactions is the genetic variability of each individual that confers a differential susceptibility to each insult, adding another layer of complexity. However, all of these insults can cause insulin resistance, albeit via different mechanisms.

We propose that there may be multiple factors contributing to insulin resistance in an individual. We aim to identify a "signature" or "profile" for each of the causative agents of insulin resistance. Profiling of patients could then allow the determination of which subtypes of insulin resistance each individual has. One such subtype of insulin resistance is that caused by increased saturated fatty acid levels in some obese individuals. We hypothesise that we can use the profiles to identify a main contributing subtype to a patient's insulin resistance. Then we will aim to specifically target that subtype (or subtypes) in an individual for longer term and personalised management of their metabolic dysregulation. This will be discussed in further detail below.

\subsection{Obesity}

The most commonly associated disorder linked with the onset of insulin resistance is obesity (Cummings and Schwartz, 2003; Granberry and Fonseca, 1999). Obesity is widespread in the western world, with the recent US National Health and Nutrition Examination Survey 
(NHANES) finding that $67 \%$ of Americans aged 20 and above are overweight or obese, with $34 \%$ being obese (NCHS, 2008). The WHO estimates that in 2005 there were 1.6 billion adults worldwide who were overweight, at least 400 million of who were obese. These numbers are projected to increase to 2.3 billion overweight and at least 700 million obese adults by 2015 (WHO, 2006). The increasing epidemic of obesity will further increase the prevalence of insulin resistance and T2D within society, making the development of effective treatments a critical challenge for the $21^{\text {st }}$ century.

As one of the primary risk factors for the development of T2D, obesity warrants extensive study as a target for the development of additional and alternative therapies. The defining characteristic of obesity is increased adiposity. Increased availability of free fatty acids (FFA) in patients with obesity plays a critical role in the development of insulin resistance (DeFronzo, 2004). There are numerous factors in obesity which can lead to increases in circulating free fatty acids, including exceeding the storage capacity of adipose tissue by excess caloric intake (Langeveld and Aerts, 2009), and adipose tissue stimulation by the paracrine tumour necrosis factor alpha (TNFa) which induces triglyceride metabolism and free fatty acid release (Ruan and Lodish, 2003). Insulin resistance in adipose tissue can also lead to excess fatty acid release, due to suppression of the antilipolytic effects of insulin (Ruan and Lodish, 2003). The direct effects of increased circulating free fatty acids on macrophages to stimulate release of pro-inflammatory cytokines such as TNFa and IL-6 has also been recently described (for a review see (Bilan et al., 2009)). The onset of insulin resistance caused by free fatty acids is therefore highly complex, and although direct action upon target tissues have been described, there are secondary actions upon other tissue types which further complicate the pathology of the disease. Given the increasing prevalence of obesity around the world, dissecting the mechanisms by which free fatty acids contribute to insulin resistance may identify new avenues for effective treatment regimes

\section{Previous approaches at characterising insulin resistance}

\subsection{Classical single target-based approaches}

Classical approaches for dissecting insulin resistance involve targeting signalling defects in both in vitro and in vivo models of insulin resistance. These approaches - including western blotting for proteins and PCR for genetic data have enhanced our knowledge of insulin resistance and the mechanisms by which insulin signalling is impaired. However such approaches rely upon previous knowledge to build a network of signalling connections, and implicating signalling defects in the observed in vitro or in vivo model being observed. As is becoming increasingly clear, signalling networks within cells are far more complicated than previously thought. Single insults (such as fatty acids) not only impact upon insulin signalling directly, but also on numerous signalling cascades such as inflammatory pathways, which may be either distally related to insulin signalling or not related at all. This will result in activation of many kinases, and modification of transcription of a number of genes, in the process of the cell reaching an equilibrium state.

We now know that the single target or pathway approaches provide too narrow a window to appreciate the changes induced in complex disease states. While the contribution of the single target / pathway approaches cannot be denied, in terms of expanding our knowledge base, a wider approach is now required for the development of the next line of therapies. 


\subsection{Endpoint-based approaches}

Endpoint-based approaches have been significant in developing our understanding of the development of diabetes. Utilising insulin signalling endpoints such as hepatic glucose output or muscle glucose transport can provide a more global overview of the cellular state compared with the phosphorylation of a single kinase amongst a signalling network. The discovery of new therapies targeted against endpoints allow us to bypass the upstream complexity that hinders the target-based approaches.

\section{3 'Omics' approaches}

The development of powerful platform technologies such as microarrays has led to a vast increase in the utilisation of the 'omics' type studies. Current mass spectroscopy techniques allow for the study of nearly the entire lipid or protein fraction of a sample, allowing characterisation of disease states in an unprecedented way. The requirement to investigate and treat many diseases with multifactorial natures has necessitated the need for more powerful technologies to give researchers a "global" view of disease states. The search for effective early diagnostic tools, insight into the development of disease states, and the development of new therapies are increasingly relying on one or more of these new platform approaches.

In the context of obesity, lipidomic approaches are proving to be very useful in identifying characteristic changes in tissue-specific lipid profiles of patients with T2D (Meikle and Christopher, 2011), which has been made possible by advances in mass spectroscopy techniques. Advances have also been applied to the proteomic field. Techniques such as Stable Isotope Labeling by Amino acids in Cell culture (SILAC) are proving to be powerful in furthering our knowledge of insulin signalling cascades in both normal and insulin resistant states, by allowing the investigation of a large number of proteins at once across multiple samples (Hanke and Mann, 2009).

\subsection{Genomics-based approaches}

Developed in the mid 90's for the analysis of the expression of multiple genes in parallel (Schena et al., 1995), microarray technology can now be used to assess the expression of tens of thousands of genes in a sample simultaneously. This provides a powerful tool to assess whole cell transcriptional events for any given cell or tissue in any biological state. Microarray technology has a range of applications including identifying disease-causing genes, identifying targets for new therapies and prediction of drug responsiveness (Jayapal and Melendez, 2006).

Two major applications for microarray technology involve examining gene sets for pathway analysis, and examining differentially expressed genes between two or more experimental conditions (Kauffmann and Huber, 2010). Gene set enrichment analysis (GSEA) involves taking a gene list, ranked according to the difference in expression between the phenotypes or treatments being investigated. The goal of GSEA is to determine whether members of specific gene sets (grouped on functional similarity), are ranked together towards the top or bottom of the list. GSEA therefore indicates whether a correlation exists between differential expression of that set of genes, and the specific phenotype being investigated (Oron et al., 2008; Subramanian et al., 2005). This pathway analysis approach to dissecting disease is complimented well by proteomic approaches which can similarly be used for pathway analysis. 
The second of the two applications involves performing microarray analysis on gene sets from multiple experimental conditions, and can be used to identify differentially expressed genes in differing disease states. This 'shotgun' style approach to genome analysis can yield previously unknown information about the regulation of disease states at the transcriptional level, which can have important implications for understanding the pathophysiology of disease. The set of differentially expressed genes can also be used for a diagnostic approach to the disease. Applying Bayesian Linear statistical modelling to gene sets allows for selection of a relatively small gene set which can characterise the particular biological state of the cell or tissue being investigated (Smyth, 2004). This process statistically evaluates which set of genes have the greatest differential expression between the conditions tested, and identifies a 'fingerprint' indicative of the biological state of the cell or tissue involved, known as a gene expression signature (GES). Previously, GESs have been applied to the field of cancer research, for applications such as classifying tumour types and predicting tumour response to chemotherapy. By classifying tumours into distinct types, and with knowledge of how each type will respond to particular therapies, clinicians are therefore able to treat patients more effectively by personalising treatment regimes (Lee et al., 2007). Personalised medicine approaches such as this are becoming increasingly important tools in fighting diseases and the use of GES are likewise increasing in disease research.

\section{Gene expression signatures}

\subsection{Gene expression signatures as a diagnostic tool}

First described in 2000, GESs were developed in the field of cancer research. The differences in patient response to therapies led researchers to believe that groups of cancers that were not able to be histologically characterised were actually a heterogeneous group of tumours. Seeking a non-biased method for classification, gene expression data was investigated to search for patterns which could differentiate classes of B-cell lymphomas with differing patient survival rates (Alizadeh et al., 2000). The main outcome of the study was the finding of two subgroups, classified on the basis of differential gene expression of hundreds of genes, with differing survival outcomes for patients. This early study was instrumental in highlighting the use of gene expression data as a disease classification tool. The power of the GES approach is that entire genome datasets are narrowed down to the smallest number of genes capable of robustly characterising differences between biological samples. Using complex statistical analysis of large datasets, the prediction power of these small subsets of genes has been shown to be equivalent to the whole dataset. Once developed, the GES tool allows for rapid, reliable characterisation of various cellular states, which has a number of important applications.

Accurate classification of disease states plays a vital role in diagnosis and treatment. GESs have been successfully used in a number of different cancer types including breast (Nuyten et al., 2008; van de Vijver et al., 2002), gastric (Cui et al., 2011), lung, colon and ovarian cancer (Mettu et al., 2010) to aid in prediction of survival, and to guide clinicians in choice of treatments for their patients. Recently, GESs have even been applied to predicting the likelihood of side effects in patients treated by acute radiotherapy (Mayer et al., 2011). Using GES technology for prediction and/or classification however represents only part of its potential. The use of GESs for the discovery and development of new therapies is perhaps the most promising application of this technology. The use of GESs to develop new therapies is especially powerful when a specific endpoint is known, but intermediate signalling steps or the molecular targets have not been identified. Provided a model for the disease of interest has 
been developed, high throughput screening of small molecule libraries can be performed by assessing the effects of those agents on the mRNA levels of the genes identified as the GES. The GES approach has been used in a number of cancer models to identify new therapies, which have increased efficacy over current treatments. For acute myelogenous leukemias, the identification of inducers of terminal differentiation has opened up new therapeutic avenues previously unavailable (Stegmaier et al., 2004). For the treatment of Ewing sarcomas, the targeting of the EWS/FLI oncoprotein had previously been unsuccessful with screening approaches, until the GES approach was used successfully to identify cytosine arabinoside as a modulator of the EWS/FLI oncoprotein (Stegmaier et al., 2007).

What makes GESs unique is that the GES genes are not limited to genes known to be involved in the particular physiological process being investigated. A GES is the minimal set of genes that best defines the difference between two biological samples - be that a disease state or the physiological response to a particular drug or chemical. While it is possible that a GES gene plays a role in the specific model being investigated, it is also possible it does not, and thus any conclusions based upon the identity of genes in the GES must be confirmed with subsequent studies.

\section{Overview of GES development}

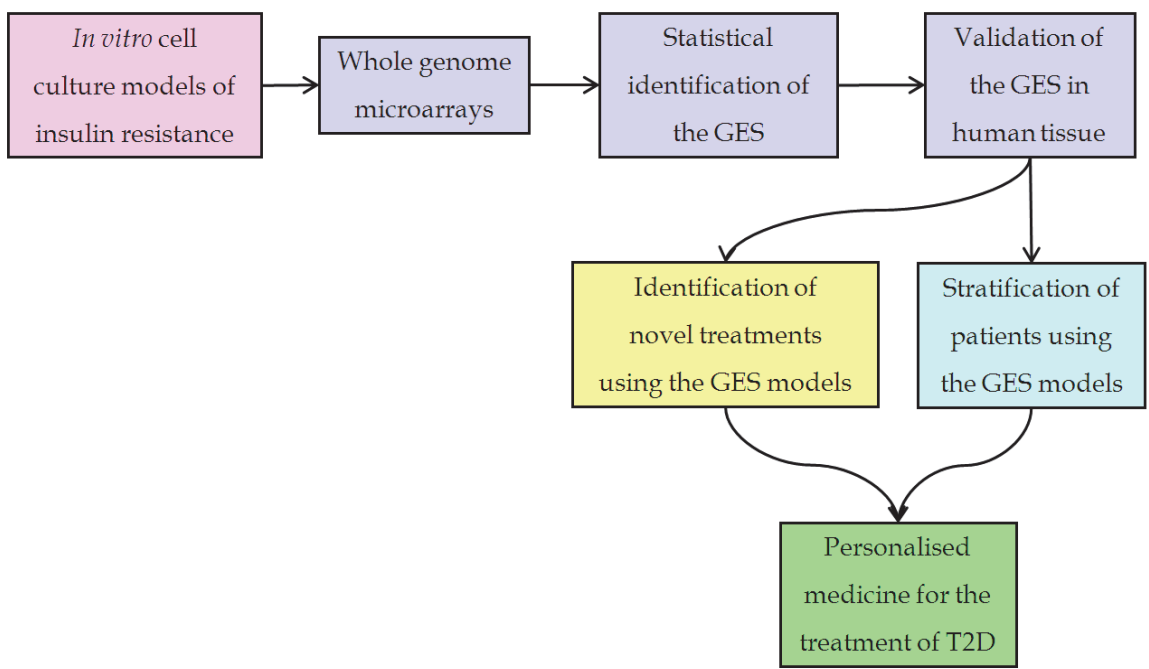

Fig. 3. Development procedure for a GES for insulin resistance, based upon (Stegmaier et al., 2004). mRNA is extracted from in vitro cell culture models of insulin resistance, and analysed using whole genome microarrays. The GES genes are identified, and validated in human tissues. The validated GES can then be used to identify novel treatments, as well as stratify patients. This allows for the personalised treatment of T2D.

\subsection{Application to dissecting insulin resistance subtypes}

We propose that GESs can be applied to dissect and study insulin resistance subtypes. The GES methodology described here can be undertaken in either animal tissues or cell culture models. Due to the high reproducibility required when extracting the data from relevant 
platform technology (for example, microarray), we have found that working in cell culture systems is the most robust and consistent approach. Once the GES is developed from a cell culture model, the biological relevance of an in vitro-derived GES requires validation in human tissue. Validation of the GES in human cohorts tests for a correlation between the homeostasis model assessment (HOMA) measures of insulin resistance, based upon plasma glucose and insulin levels (Matthews et al., 1985), and similarity to the GES profile for each subject. If it can be shown that those patients whose expression profiles were most similar to the GES showed a greater degree of insulin resistance as indicated by the HOMA score, the GES is considered to be valid in human tissue. The modelling of insulin resistance subtypes in the GES models involves the use of a specific insult to induce insulin resistance which are known to cause insulin resistance in individuals. Such insults include saturated fatty acids (PA) or mediators of chronic inflammation (TNFa).

The development of a GES in cell culture requires modelling three distinct cellular states relating to insulin sensitivity. The first state is that of a 'healthy', insulin sensitive cell. The second state is that of a 'diseased', insulin resistant cell. This is achieved by treatment of the target cells with the insulin resistance insult such as TNFa or PA. The third state represents a 'recovered from disease' state, which is achieved by treating insulin resistant cells with a cocktail of antidiabetic agents to restore insulin action. The definition of these three states is deliberate and critical to the integrity of the GES. Insulin resistance in this model system is measured using a key endpoint of insulin action, such as glucose uptake in muscle or adipose tissue, or glucose production in the liver, and will be discussed in further detail below (see section 6.2).

To apply the GES approach to insulin resistance, firstly we assessed the significant changes in gene expression levels between the 'healthy' insulin-responsive cells, and the 'insulin resistant' cells to identify the genes which change in response to the insulin resistance-inducing insult. In order to determine which genes are being affected due to insulin resistance and not nonspecific changes induced by the insult per se, the changes induced by the 'recovered from disease' state was then assessed. Only those genes whose expression levels were significantly changed in the 'diseased' state, and then changed again in the reverse direction in the 'recovered from disease' state are used for the development of the GES. It is this group of genes whose expression are linked to the insulin resistant state of the cell or tissue.

\subsection{Characterising insulin resistance in vitro}

In order to effectively model insulin resistance in vitro, an endpoint measure of insulin action is required. Cell-based models offer a number of assays which can be used to determine insulin signalling in both sensitive and insulin resistant states. In vitro models of insulin resistance can be developed in each of the main insulin sensitive tissues; muscle, adipose and liver. One key measure of insulin action in muscle and adipose cells is glucose uptake. In liver cells, regulation of gluconeogenesis by insulin is one of the key endpoints of insulin action. These assays work by measuring the relevant endpoint (glucose uptake or gluconeogenesis) in the presence and absence of an insulin resistance insult to characterise insulin resistance. As the in vitro cell culture model must be manipulated from healthy to diseased and then restored, a robust and large dynamic range is needed in the bioassay used to measure the insulin resistance endpoint parameter.

Reversal of insulin resistance involves assessing a wide range of known insulin sensitisers in the model of choice. A combination therapy which is able to fully reverse insulin resistance is selected, based upon its ability to not only reverse insulin resistance, but also avoid negatively 
impacting upon cellular viability. Combination therapy is required, as this will ensure that the GES is characteristic of an insulin resistant state which has been reversed by a multi-target approach. There is a greater chance that in drug development the GES will identify novel therapies, rather than the individual therapies used in its creation - as may happen with a single treatment GES. Potential reversers of insulin resistance include known antidiabetic drugs such as the biguanide metformin, TZDs, chemical chaperones such as tauroursodeoxycholic acid (TUDCA) (Iglesias et al., 2002), antioxidants such as N-acetylcysteine (NAC) (Houstis et al., 2006), and NSAIDs such as aspirin (Sinha et al., 2004; Yuan et al., 2001).

\subsubsection{Personalised treatment for patients}

The GES holds promise for personalised treatments for patients by allowing the stratification of patients based on subgroups of insulin resistance. Once patients are sub grouped, treatments can be personalised to their individual diagnosis, leading to improved health outcomes. The subgrouping of patients according to the GES involves measuring the expression levels of the GES genes in the patient. Regardless of which tissue or cell type the GES is derived from, a non-invasive, easy to obtain sample is needed to facilitate screening of as many individuals as possible. A blood sample is ideal for these requirements. Lymphocyte gene expression profiles have been shown to correlate well with gene expression profiles of insulin responsive tissues including liver and adipose tissue (Iida et al., 2006). We propose that by measuring the expression levels of the GES genes in a patients white blood cells we can subtype patients according to one or more GES. The GES which best correlates with the gene expression pattern of a patient's white blood cells will therefore indicate a specific avenue of treatment for that patient (see Fig. 4).

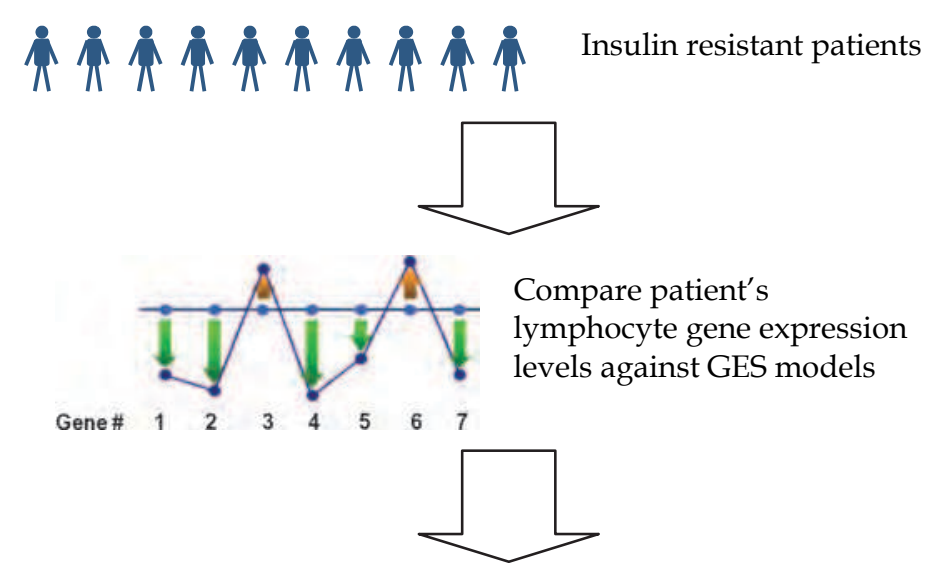

Stratify patients on a continuum

Highest similarity group $\rightarrow$ Lowest similarity group according to their similarity to one of the GES models $\rightarrow$ appropriate treatment regime

Fig. 4. Stratification of patients according to their similarities to the GES models of insulin resistance. 


\subsubsection{Development of "targeted" therapies}

The GES can be used to aid in the development of new therapies for T2D, by allowing for high throughput screening for new drugs with insulin sensitising and antidiabetic properties. Screening involves treating cells with chemical libraries, which can include previously known and marketed drugs. After screening the GES genes in the treated cells, the key analysis is comparing the GES genes in the treated cells with the GES profile of the specific model being used. Those chemicals which mimic the GES profile of successful reversal of insulin resistance are identified as the most promising candidate drugs. These drugs can then be validated both in vitro and in vivo to assess their efficacy. We propose that new therapies identified via this approach may show increased efficacy in treating patients subtyped by the same gene expression signature. The subtyping of patients according to gene expression signatures, as well as the potential for targeted therapies against each subtype, represents a personalised medicine approach for the treatment of insulin resistance and T2D.

\subsection{Proof of principle: Inflammation-induced cellular "insulin resistance"}

As proof of principle, we recently developed a GES for TNFa-induced insulin resistance (Konstantopoulos et al., 2011). Using 3T3-L1 adipocytes as the cell-based model, we identified 3325 genes whose expression was altered by the induction of insulin resistance by TNFa. Of those genes, only 1022 showed altered expression by the reversal of insulin resistance with the insulin sensitisers aspirin and troglitazone. From those 1022 genes, a set of 5 genes were selected whose combined expression profile gave the highest predictive power to differentiate the insulin resistant state, and the re-sensitised state.

As described above, GESs can be used for screening of patients with T2D. We evaluated this by assessing whether the in vitro-derived GES for TNFa could characterise insulin resistant subtypes in a human cohort. We used lymphocytes from the San Antonio Family Heart Study (Mitchell et al., 1996), and measured the expression of 47,289 transcripts in 1,240 individuals from 42 extended families (Goring et al., 2007). The TNFa GES of 5 genes was detected in the human profile dataset, and GES score assigned - comprising the sum of the absolute values of the standardised expression units of each of the 5 genes. This was tested for association with HOMA measures of insulin resistance for each subject. Those patients whose expression profiles were most similar to the TNFa GES showed a higher degree of insulin resistance as indicated by the HOMA score ( $<<0.001)$ (Konstantopoulos et al., 2011). This correlation is consistent with the use of GES technology to characterise an insulin resistant subtype in this population.

In vitro screening of compound libraries has also been used in this model, assessing the ability of a given compound to affect the genes identified in the GES (Fig. 5). Screening for those compounds whose effects on the target genes mirrored the expression profile observed in the gene expression signature was successful in identifying known and novel insulin sensitising agents such as non-steroidal anti-inflammatory agents, $\beta$-adrenergic antagonists, beta-lactams and sodium channel blockers (Konstantopoulos et al., 2011).

Investigation of the GES genes, and their role in insulin resistance has also yielded positive outcomes. We conducted a series of studies to assess what role (if any) the GES genes might play in the development of insulin resistance. Our investigation of the GES gene STEAP4 was mirrored by the results of data published at that time which showed that STEAP4 protects against inflammation and metabolic dysfunction (Wellen et al., 2007). This 
highlighted the utility of the GES in gene discovery related to the particular biological state being investigated, and is further proof of the power of this technique in investigating disease states.

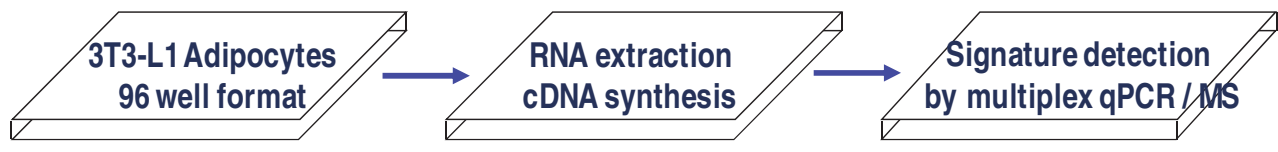

Insulin Resistant

$3 \mathrm{ng} / \mathrm{ml}$ TNF $\alpha$ for $72 \mathrm{hr}$

Compound Screen

$10 \mu \mathrm{M}$ each compound

for last $24 \mathrm{hr}$

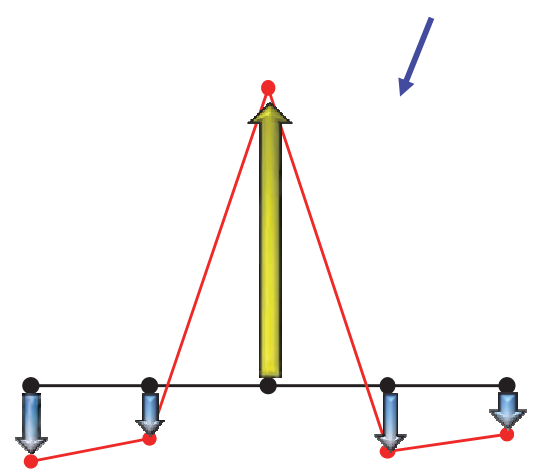

Normalised gene expression levels in the 'insulin resistant'state Gene expression levels in the 'insulin re-sensitised'state

Fig. 5. GES screen of a chemical library. Screening involves assessing the effects of compounds on the GES profile in insulin resistant cells.

\subsection{Identification of palmitate-derived GES from liver cells}

Following the development of the TNFa GES, a GES for palmitic acid (PA) induced insulin resistance is currently being developed. The cell model has been established in FAO liver cells, with insulin resistance achieved after incubating the cells with $75 \mu \mathrm{M}$ PA for $48 \mathrm{~h}$. This insulin resistant phenotype has been reversed by treating PA treated cells with $0.25 \mathrm{mM}$ metformin and $2 \mathrm{mM}$ sodium salicylate (NAS) in the final 24 hours of PA incubation (Fig. 6). This model has been developed using the same statistical modelling as the TNFa GES. The identity of the GES genes for this model is currently being determined. 


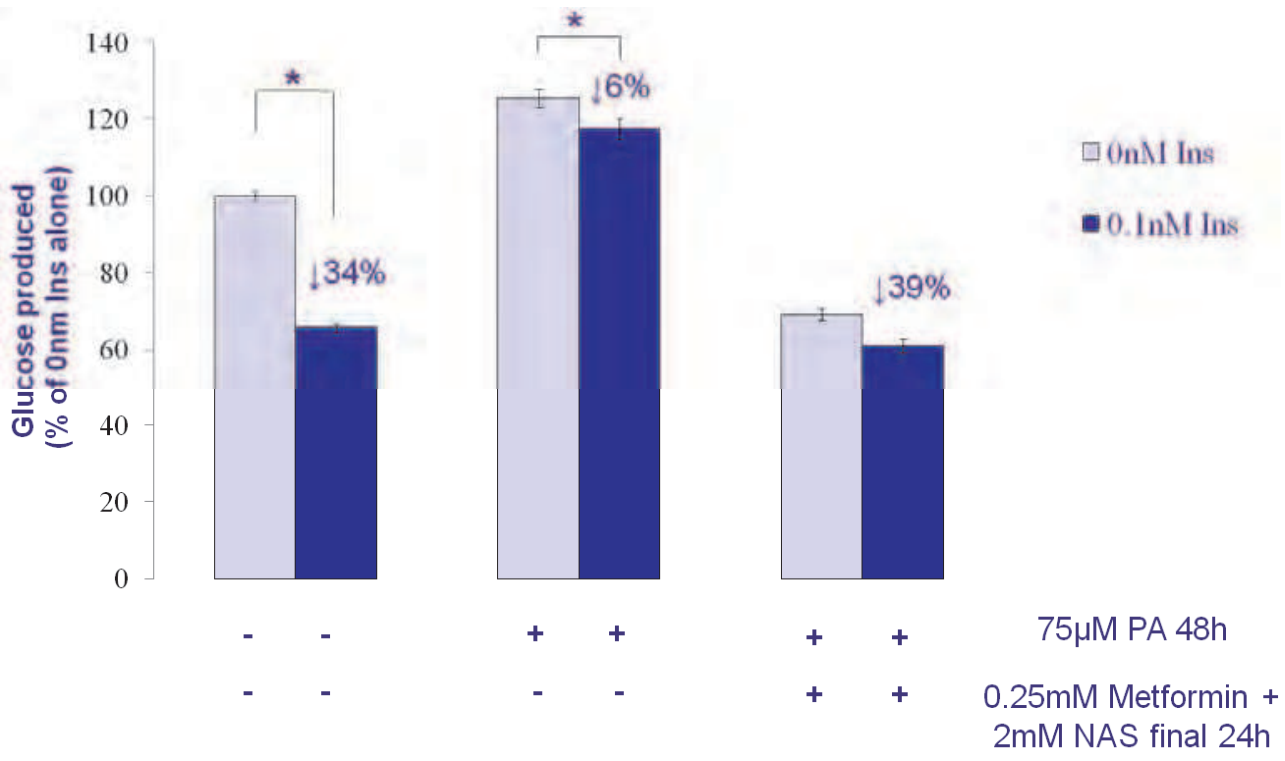

Fig. 6. PA induced insulin resistance in FAO liver cells. Insulin stimulation of FAO hepatocytes decreased glucose production by $34 \pm 1 \%\left({ }^{*}, \mathrm{p} \leq 0.005\right.$ compared with basal cells, $\mathrm{n}=8$ ). Exposure of FAO hepatocytes to $75 \mu \mathrm{M}$ PA for $48 \mathrm{~h}$ decreased insulin-induced suppression of glucose production to only $6 \pm 3 \%\left({ }^{*}, \mathrm{p} \leq 0.005\right.$ compared with PA-treated, basal hepatocytes, $\mathrm{n}=8$ ). Addition of $0.25 \mathrm{mM}$ metformin and $2 \mathrm{mM}$ NAS in the final $24 \mathrm{~h}$ of the $48 \mathrm{~h}$ PA treatment reversed this increase in glucose production, reducing insulinstimulated glucose release to the same levels as observed in vehicle treated cells.

The PA derived GES will be used for the stratification of patient cohorts as described above. We anticipate that the PA derived GES will identify an insulin resistant subpopulation from the cohorts we test it in. A key comparison with the different GES models will be the identity of the subgroups identified, and the degree of overlap (if any) observed in the groups. Drug screening, as well as investigation of the GES genes will also be performed for the PA derived GES.

\section{Conclusion}

The use of 'omics' style approaches to disease states such as T2D are becoming increasingly accepted as one way research should investigate these diseases in the $21^{\text {st }}$ century. The success of GES technology in the cancer field as both a diagnostic tool and a drug discovery tool is becoming increasingly apparent, and we have shown this technology is equally applicable to the study of T2D. As disease research is progressing towards the development of personalised medicine as the 'holy grail' for treatment regimes, we foresee a future where personalised medicine is seen as the gold standard for patient care. We believe GES technology will provide a platform for the development of novel, personalised treatments for patients with T2D. 


\section{Acknowledgement}

The authors wish to thank Juan Molero for his advice and assistance in the development of the hepatic model of PA induced insulin resistance.

\section{References}

Alizadeh, A.A., Eisen, M.B., Davis, R.E., Ma, C., Lossos, I.S., Rosenwald, A., Boldrick, J.C., Sabet, H., Tran, T., Yu, X., et al. (2000). Distinct types of diffuse large B-cell lymphoma identified by gene expression profiling. Nature 403, 503-511.

Baudry, A., Leroux, L., Jackerott, M., and Joshi, R.L. (2002). Genetic manipulation of insulin signaling, action and secretion in mice. Insights into glucose homeostasis and pathogenesis of type 2 diabetes. EMBO Rep 3, 323-328.

Bergman, R.N., and Ader, M. (2000). Free fatty acids and pathogenesis of type 2 diabetes mellitus. Trends Endocrinol Metab 11, 351-356.

Bilan, P.J., Samokhvalov, V., Koshkina, A., Schertzer, J.D., Samaan, M.C., and Klip, A. (2009). Direct and macrophage-mediated actions of fatty acids causing insulin resistance in muscle cells. Arch Physiol Biochem 115, 176-190.

CDC (2011). National diabetes fact sheet: national estimates and general information on diabetes and prediabetes in the United States, C.f.D.C.a.P. U.S. Department of Health and Human Services, ed. (Atlanta).

Chavez, J.A., and Summers, S.A. (2003). Characterizing the effects of saturated fatty acids on insulin signaling and ceramide and diacylglycerol accumulation in 3T3-L1 adipocytes and C2C12 myotubes. Arch Biochem Biophys 419, 101-109.

Cohen, A., and Horton, E.S. (2007). Progress in the treatment of type 2 diabetes: new pharmacologic approaches to improve glycemic control. Curr Med Res Opin 23, 905-917.

Cui, J., Li, F., Wang, G., Fang, X., Puett, J.D., and Xu, Y. (2011). Gene-expression signatures can distinguish gastric cancer grades and stages. PLoS ONE 6, e17819.

Cummings, D.E., and Schwartz, M.W. (2003). Genetics and pathophysiology of human obesity. Annu Rev Med 54, 453-471.

Davies, M.J., Raymond, N.T., Day, J.L., Hales, C.N., and Burden, A.C. (2000). Impaired glucose tolerance and fasting hyperglycaemia have different characteristics. Diabetic Medicine 17, 433-440.

DeFronzo, R.A. (2004). Pathogenesis of type 2 diabetes mellitus. Med Clin North Am 88, 787-835, ix.

Drucker, D.J. (2003). Glucagon-like peptide-1 and the islet beta-cell: Augmentation of cell proliferation and inhibition of apoptosis. Endocrinology 144, 5145-5148.

Drucker, D.J. (2005). Biologic actions and therapeutic potential of the proglucagon-derived peptides. Nat Clin Pract Endocrinol Metab 1, 22-31.

Dunstan, D.W., Zimmet, P.Z., Welborn, T.A., De Courten, M.P., Cameron, A.J., Sicree, R.A., Dwyer, T., Colagiuri, S., Jolley, D., Knuiman, M., et al. (2002). The rising prevalence of diabetes and impaired glucose tolerance: the Australian Diabetes, Obesity and Lifestyle Study. Diabetes Care 25, 829-834.

Gavin, J.R., Alberti, K.G., Davidson, M.B., DeFronzo, R.A., Drash, A., Gabbe, S.G., Genuth, S., Harris, M., Kahn, R., Keen, H., et al. (2003). Report of the expert committee on the diagnosis and classification of diabetes mellitus. Diabetes Care 26 Suppl 1, S5-20. 
GlaxoSmithKline (2010). GlaxoSmithKline Annual Report 2010 (Brentford, United Kingdom, GlaxoSmithKline).

Goring, H.H.H., Curran, J.E., Johnson, M.P., Dyer, T.D., Charlesworth, J., Cole, S.A., Jowett, J.B.M., Abraham, L.J., Rainwater, D.L., Comuzzie, A.G., et al. (2007). Discovery of expression QTLs using large-scale transcriptional profiling in human lymphocytes. Nature Genetics 39, 1208-1216.

Granberry, M.C., and Fonseca, V.A. (1999). Insulin resistance syndrome: options for treatment. South Med J 92, 2-15.

Groop, L.C. (1992). Sulfonylureas in NIDDM. Diabetes Care 15, 737-754.

Hanke, S., and Mann, M. (2009). The phosphotyrosine interactome of the insulin receptor family and its substrates IRS-1 and IRS-2. Mol Cell Proteomics 8, 519-534.

Houstis, N., Rosen, E.D., and Lander, E.S. (2006). Reactive oxygen species have a causal role in multiple forms of insulin resistance. Nature 440, 944-948.

Iglesias, M.A., Ye, J.M., Frangioudakis, G., Saha, A.K., Tomas, E., Ruderman, N.B., Cooney, G.J., and Kraegen, E.W. (2002). AICAR administration causes an apparent enhancement of muscle and liver insulin action in insulin-resistant high-fat-fed rats. Diabetes 51, 2886-2894.

Iida, S., Sato, Y., Nakaya, A., Shinohara, Y., Hayashi, Y., Sawada, A., Nagata, H., Kaji, N., Kamiya, H., Baba, Y., et al. (2006). Genome wide expression analysis of white blood cells and liver of pre-diabetic Otsuka Long-Evans Tokushima Fatty (OLETF) rats using a cDNA microarray. Biological \& Pharmaceutical Bulletin 29, 2451-2459.

Itani, S.I., Ruderman, N.B., Schmieder, F., and Boden, G. (2002). Lipid-Induced Insulin Resistance in Human Muscle Is Associated With Changes in Diacylglycerol, Protein Kinase C, and II $^{\circ}$ B-Ît. Diabetes 51, 2005-2011.

Jayapal, M., and Melendez, A.J. (2006). DNA microarray technology for target identification and validation. Clin Exp Pharmacol Physiol 33, 496-503.

Jove, M., Planavila, A., Sanchez, R.M., Merlos, M., Laguna, J.C., and Vazquez-Carrera, M. (2006). Palmitate induces tumor necrosis factor-alpha expression in C2C12 skeletal muscle cells by a mechanism involving protein kinase $C$ and nuclear factor-kappaB activation. Endocrinology 147, 552-561.

Kauffmann, A., and Huber, W. (2010). Microarray data quality control improves the detection of differentially expressed genes. Genomics.

Kendall, D.M., Riddle, M.C., Rosenstock, J., Zhuang, D.L., Kim, D.D., Fineman, M.S., and Baron, A.D. (2005). Effects of exenatide (exendin-4) on glycemic control over 30 weeks in patients with type 2 diabetes treated with metformin and a sulfonylurea. Diabetes Care 28, 1083-1091.

Knowler, W.C., Barrett-Connor, E., Fowler, S.E., Hamman, R.F., Lachin, J.M., Walker, E.A., and Nathan, D.M. (2002). Reduction in the incidence of type 2 diabetes with lifestyle intervention or metformin. N Engl J Med 346, 393-403.

Konstantopoulos, N., Foletta, V.C., Segal, D.H., Shields, K.A., Sanigorski, A., Windmill, K., Swinton, C., Connor, T., Wanyonyi, S., Dyer, T.D., et al. (2011). A Gene Expression Signature for Insulin Resistance. Physiol Genomics 43, 110-120.

Kristensen, J.S., Frandsen, K.B., Bayer, T., and Muller, P.G. (2000). Compared with repaglinide sulfonylurea treatment in type 2 diabetes is associated with a 2.5 -fold increase in symptomatic hypoglycemia with blood glucose levels $<45 \mathrm{mg} / \mathrm{dl}$. Diabetes 49, A131-A131. 
Langeveld, M., and Aerts, J.M. (2009). Glycosphingolipids and insulin resistance. Prog Lipid Res 48, 196-205.

Lee, J.K., Havaleshko, D.M., Cho, H., Weinstein, J.N., Kaldjian, E.P., Karpovich, J., Grimshaw, A., and Theodorescu, D. (2007). A strategy for predicting the chemosensitivity of human cancers and its application to drug discovery. Proc Natl Acad Sci U S A 104, 13086-13091.

Lillioja, S., Mott, D.M., Spraul, M., Ferraro, R., Foley, J.E., Ravussin, E., Knowler, W.C., Bennett, P.H., and Bogardus, C. (1993). Insulin-Resistance and Insulin Secretory Dysfunction as Precursors of Non-Insulin-Dependent Diabetes-Mellitus Prospective Studies of Pima-Indians. New England Journal of Medicine 329, 19881992.

Matthews, D.R., Hosker, J.P., Rudenski, A.S., Naylor, B.A., Treacher, D.F., and Turner, R.C. (1985). Homeostasis model assessment: insulin resistance and beta-cell function from fasting plasma glucose and insulin concentrations in man. Diabetologia 28, 412-419.

Mayer, C., Popanda, O., Greve, B., Fritz, E., Illig, T., Eckardt-Schupp, F., Gomolka, M., Benner, A., and Schmezer, P. (2011). A radiation-induced gene expression signature as a tool to predict acute radiotherapy-induced adverse side effects. Cancer Letters 302, 20-28.

McKinlay, J., and Marceau, L. (2000). US public health and the 21st century: diabetes mellitus. Lancet 356, 757-761.

Meikle, P.J., and Christopher, M.J. (2011). Lipidomics is providing new insight into the metabolic syndrome and its sequelae. Curr Opin Lipidol.

Mettu, R.K.R., Wan, Y.W., Habermann, J.K., Ried, T., and Guo, N.L. (2010). A 12-gene genomic instability signature predicts clinical outcomes in multiple cancer types. Int J Biol Marker 25, 219-228.

Mitchell, B.D., Kammerer, C.M., Blangero, J., Mahaney, M.C., Rainwater, D.L., Dyke, B., Hixson, J.E., Henkel, R.D., Sharp, R.M., Comuzzie, A.G., et al. (1996). Genetic and environmental contributions to cardiovascular risk factors in Mexican Americans. The San Antonio Family Heart Study. Circulation 94, 2159-2170.

Mlinar, B., Marc, J., Janez, A., and Pfeifer, M. (2007). Molecular mechanisms of insulin resistance and associated diseases. Clin Chim Acta 375, 20-35.

Nathan, D.M., Buse, J.B., Davidson, M.B., Heine, R.J., Holman, R.R., Sherwin, R., and Zinman, B. (2006). Management of hyperglycemia in type 2 diabetes: A consensus algorithm for the initiation and adjustment of therapy: a consensus statement from the American Diabetes Association and the European Association for the Study of Diabetes. Diabetes Care 29, 1963-1972.

NCHS (2008). Prevalence of overweight, obesity and extreme obesity among adults: United States, trends 1976-80 through 2005-2006 N.C.f.H.S. (US), ed.

NIDDK (2009). National Diabetes Information Clearinghouse (NDIC).

Nissen, S.E., and Wolski, K. (2010). Rosiglitazone Revisited: An Updated Meta-analysis of Risk for Myocardial Infarction and Cardiovascular Mortality. Arch Intern Med.

Nuyten, D.S., Hastie, T., Chi, J.T., Chang, H.Y., and van de Vijver, M.J. (2008). Combining biological gene expression signatures in predicting outcome in breast cancer: An alternative to supervised classification. Eur J Cancer 44, 2319-2329. 
Oron, A.P., Jiang, Z., and Gentleman, R. (2008). Gene set enrichment analysis using linear models and diagnostics. Bioinformatics 24, 2586-2591.

Potes, C.S., and Lutz, T.A. (2010). Brainstem mechanisms of amylin-induced anorexia. Physiology \& Behavior 100, 511-518.

Ruan, H., and Lodish, H.F. (2003). Insulin resistance in adipose tissue: direct and indirect effects of tumor necrosis factor-alpha. Cytokine Growth Factor Rev 14, 447-455.

Salpeter, S., Greyber, E., Pasternak, G., and Salpeter, E. (2006). Risk of fatal and nonfatal lactic acidosis with metformin use in type 2 diabetes mellitus. Cochrane Database of Systematic Reviews, - .

Saltiel, A.R., and Pessin, J.E. (2002). Insulin signaling pathways in time and space. Trends Cell Biol 12, 65-71.

Schena, M., Shalon, D., Davis, R.W., and Brown, P.O. (1995). Quantitative monitoring of gene expression patterns with a complementary DNA microarray. Science 270, 467470 .

Schmitz, O., Brock, B., and Rungby, J. (2004). Amylin agonists: a novel approach in the treatment of diabetes. Diabetes 53 Suppl 3, S233-238.

Sharma, A.M. (2006). The obese patient with diabetes mellitus: from research targets to treatment options. Am J Med 119, S17-23.

Sinha, S., Perdomo, G., Brown, N.F., and O'Doherty, R.M. (2004). Fatty acid-induced insulin resistance in L6 myotubes is prevented by inhibition of activation and nuclear localization of nuclear factor kappa B. J Biol Chem 279, 41294-41301.

Smyth, G.K. (2004). Linear models and empirical bayes methods for assessing differential expression in microarray experiments. Stat Appl Genet Mol Biol 3, Article3.

Stegmaier, K., Ross, K.N., Colavito, S.A., O'Malley, S., Stockwell, B.R., and Golub, T.R. (2004). Gene expression-based high-throughput screening (GE-HTS) and application to leukemia differentiation. Nature Genetics 36, 257-263.

Stegmaier, K., Wong, J.S., Ross, K.N., Chow, K.T., Peck, D., Wright, R.D., Lessnick, S.L., Kung, A.L., and Golub, T.R. (2007). Signature-based small molecule screening identifies cytosine arabinoside as an EWS/FLI modulator in Ewing sarcoma. Plos Med 4, 702-714.

Subramanian, A., Tamayo, P., Mootha, V.K., Mukherjee, S., Ebert, B.L., Gillette, M.A., Paulovich, A., Pomeroy, S.L., Golub, T.R., Lander, E.S., et al. (2005). Gene set enrichment analysis: a knowledge-based approach for interpreting genome-wide expression profiles. Proc Natl Acad Sci U S A 102, 15545-15550.

Taylor, S.I. (1999). Deconstructing type 2 diabetes. Cell 97, 9-12.

Tisch, R., and McDevitt, H. (1996). Insulin-dependent diabetes mellitus. Cell 85, 291-297.

Unwin, N., Shaw, J., Zimmet, P., and Alberti, K.G.M.M. (2002). Impaired glucose tolerance and impaired fasting glycaemia: the current status on definition and intervention. Diabetic Medicine 19, 708-723.

van de Vijver, M.J., He, Y.D., van 't Veer, L.J., Dai, H., Hart, A.A.M., Voskuil, D.W., Schreiber, G.J., Peterse, J.L., Roberts, C., Marton, M.J., et al. (2002). A geneexpression signature as a predictor of survival in breast cancer. New England Journal of Medicine 347, 1999-2009.

Watkins, P.B. (2005). Idiosyncratic liver injury: challenges and approaches. Toxicol Pathol $33,1-5$. 
Wellen, K.E., Fucho, R., Gregor, M.F., Furuhashi, M., Morgan, C., Lindstad, T., Vaillancourt, E., Gorgun, C.Z., Saatcioglu, F., and Hotamisligil, G.S. (2007). Coordinated regulation of nutrient and inflammatory responses by STAMP2 is essential for metabolic homeostasis. Cell 129, 537-548.

WHO (2006). Obesity and Overweight: Fact Sheet \# 311 (World Health Organisation).

WHO/IDF (2006). Definition and diagnosis of diabetes mellitus and intermediate hyperglycemia (WHO/IDF).

Yki-Jarvinen, H. (2004). Thiazolidinediones. N Engl J Med 351, 1106-1118.

Yuan, M., Konstantopoulos, N., Lee, J., Hansen, L., Li, Z.W., Karin, M., and Shoelson, S.E. (2001). Reversal of obesity- and diet-induced insulin resistance with salicylates or targeted disruption of Ikkbeta. Science 293, 1673-1677. 


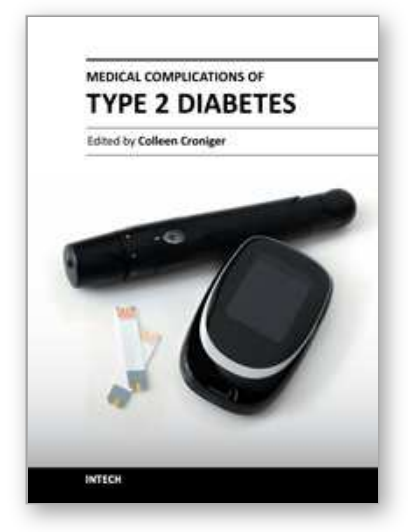

\author{
Medical Complications of Type 2 Diabetes \\ Edited by Dr. Colleen Croniger
}

ISBN 978-953-307-363-7

Hard cover, 412 pages

Publisher InTech

Published online 12, September, 2011

Published in print edition September, 2011

Obesity and type 2 diabetes are increasing worldwide problems. In this book we reviewed insulin secretion in both healthy individuals and in patients with type 2 diabetes. Because of the risk associated with progression from insulin resistance to diabetes and cardiovascular complications increases along a continuum, we included several chapters on the damage of endothelial cells in type 2 diabetes and genetic influences on endothelial cell dysfunction. Cardiovascular complications occur at a much lower glucose levels, thus a review on the oral glucose tolerance test compared to other methods was included. The medical conditions associated with type 2 diabetes such as pancreatic cancer, sarcopenia and sleep disordered breathing with diabetes were also discussed. The book concludes with several chapters on the treatments for this disease offering us hope in prevention and successful alleviation of the co-morbidities associated with obesity and type 2 diabetes.

\title{
How to reference
}

In order to correctly reference this scholarly work, feel free to copy and paste the following:

Brad Hayward, Nicky Konstantopoulos and Ken R. Walder (2011). Using Gene Expression Signatures to Dissect Insulin Resistance Subtypes, Medical Complications of Type 2 Diabetes, Dr. Colleen Croniger (Ed.), ISBN: 978-953-307-363-7, InTech, Available from: http://www.intechopen.com/books/medical-complicationsof-type-2-diabetes/using-gene-expression-signatures-to-dissect-insulin-resistance-subtypes

\section{INTECH}

open science | open minds

\author{
InTech Europe \\ University Campus STeP Ri \\ Slavka Krautzeka 83/A \\ 51000 Rijeka, Croatia \\ Phone: +385 (51) 770447 \\ Fax: +385 (51) 686166 \\ www.intechopen.com
}

\author{
InTech China \\ Unit 405, Office Block, Hotel Equatorial Shanghai \\ No.65, Yan An Road (West), Shanghai, 200040, China \\ 中国上海市延安西路65号上海国际贵都大饭店办公楼 405 单元 \\ Phone: +86-21-62489820 \\ Fax: +86-21-62489821
}


(C) 2011 The Author(s). Licensee IntechOpen. This chapter is distributed under the terms of the Creative Commons Attribution-NonCommercialShareAlike-3.0 License, which permits use, distribution and reproduction for non-commercial purposes, provided the original is properly cited and derivative works building on this content are distributed under the same license. 